\title{
Radiosensitization of esophageal carcinoma cells by the silencing of BMI-1
}

\author{
XING-XIAO YANG ${ }^{1}$, MEI-XIANG SANG ${ }^{2}$, SHU-CHAI ZHU $^{1}$, ZHI-KUN LIU $^{1}$ and MING MA ${ }^{3}$ \\ ${ }^{1}$ Department of Radiation Oncology, ${ }^{2}$ Research Centre, Department of Biotherapy, and ${ }^{3}$ Department of Clinical Laboratory, \\ The Fourth Hospital of Hebei Medical University, Shijiazhuang, Hebei 050011, P.R. China
}

Received December 4, 2015; Accepted January 8, 2016

DOI: $10.3892 / o r .2016 .4744$

\begin{abstract}
Radiotherapy (RT) has been widely used to treat cancer patients, particularly esophageal cancer patients. B-cell-specific Moloney murine leukemia virus integration site-1 (BMI-1) plays an important role in promoting the growth of cancer cells after exposure to irradiation. The present study aimed to characterize the effects of BMI-1 on the proliferation and invasion of cancer cells, as well as the mechanism involved in the regulation of the growth of esophageal cancer ECA109 and TE13 cells. The expression levels of the BMI-1 gene and protein in esophageal cancer ECA109 and TE13 cells were determined by quantitative PCR and western blotting after transfection. Co-immunoprecipitation (Co-IP) assay was employed to detect the interaction of BMI-1 with r-H2AX and H2AK119ub. We used flow cytometry to analyze the cell cycle distribution and apoptosis of transfected cells after irradiation or not, and examined cellular growth and invasion in vitro by MTS and Transwell assays. The results revealed that shRNA targeting the BMI-1 gene and protein downregulated BMI-1 expression after transfection for $24 \mathrm{~h}$. The proliferation and invasion of tumor cells in the BMI-1-shRNA group were suppressed after RT. In addition, the interaction of BMI-1, H2AK119ub and r-H2AX was increased after exposure to IR, followed by an increased apoptosis rate and decreased percentage of cells arrested at the G2/M phase after irradiation and silencing of BMI-1 by shRNA. Knockdown of BMI-1 expression decreased the phosphorylation of $\mathrm{H} 2 \mathrm{AX}$, upregulated p16, and induced the radiosensitivity of esophageal cancer ECA109 and TE13 cells in vitro and significantly inhibited the growth and invasion of tumor cells. The mechanisms were found to be abrogation of cell cycle arrest at the $\mathrm{G} 2 / \mathrm{M}$ stage and promotion of apoptosis.
\end{abstract}

Correspondence to: Professor Shu-Chai Zhu, Department of Radiation Oncology, The Fourth Hospital of Hebei Medical University, 12 Jiankang Road, Shijiazhuang, Hebei 050011, P.R. China

E-mail: sczhu1965@163.com

Key words: DNA damage response, esophageal cancer, BMI-1, radiosensitivity

\section{Introduction}

Esophageal carcinoma is a common alimentary canal malignancy with high incidence. There are $\sim 0.3104$ million new malignancies worldwide each year. Despite extensive advancement in its diagnosis and treatment, recurrence of esophageal carcinoma remains the main cause of high mortality after treatment for these patients. Current treatments, including surgical intervention, radiotherapy (RT) and chemotherapy, are moderately effective in early-stage cases, but are less effective in more advanced cases (1). For example, RT is one of the most important methods for patients at every stage of esophageal carcinoma, however, patient prognosis remains unsatisfactory and unpredictable due to profound radioresistance. Thus, identification of key molecules or pathways specifically expressed in esophageal carcinoma that are essential for the growth of cancer cells may provide novel therapeutic targets and ultimately lead to improved survival. Research over the past years clearly implicates multiple genetic alterations in the development and progression of esophageal carcinoma (2), including those that have important functions in cell adhesion, signal transduction, cell differentiation, metastasis, DNA damage and repair (3).

B-cell-specific Moloney murine leukemia virus integration site-1 (BMI-1), a member of the polycomb group of transcriptional repressors, has been detected in a variety of human carcinoma specimens, from pre-cancerous to advanced stages. In particular, BMI-1 is overexpressed in a number of malignancies $(4,5)$. Recently, it has been reported that BMI-1 is also overexpressed in alimentary canal cancers, particularly in esophageal carcinoma, which suggests that BMI-1 may confer radioresistance to esophageal carcinoma (6). Gene chip analysis also showed that BMI-1 predicts cancer metastasis (7), promotes cancer cell proliferation and invasion, causes resistance to apoptosis and enhances transfer capabilities (8). Moreover, as an active heterodimer E3 ligase, BMI-1 may induce the ubiquitination and phosphorylation of $\mathrm{H} 2 \mathrm{AX}$, which is thought to be a critical sensor that can initiate DNA damage response (DDR) (9-11). Our research has also shown that $\mathrm{H} 2 \mathrm{AX}$ is associated with the radiosensitization of esophageal cancer cells (12). However, how the function of BMI-1 in radiosensitivity is regulated remains a fundamental question that needs to be answered to elucidate the essential mechanisms controlling DDR. 
To identify the regulatory function of BMI-1 in DDR, we conducted proteomic analysis to systematically identify BMI-1 interacting proteins and the related mechanisms. However, to the best of our knowledge, the mechanisms by which BMI-1 promotes the proliferation of esophageal carcinoma cells after RT have not been reported, to date. Given the important role of BMI-1 in ionizing radiation-induced DDR, we hypothesized that silencing of BMI-1 may cause DNA damage pathway defects and thus increase radiosensitivity. In the present study, we verificated this hypothesis in vitro through two different cell lines and explored the mechanisms through which BMI-1 induces tumor progression.

\section{Materials and methods}

Cell lines and cell culture. The human esophageal squamous cell carcinoma cell lines ECA109 and TE13 were cultured in RPMI-1640 medium supplemented with $10 \%$ fetal bovine serum (FBS) at $37^{\circ} \mathrm{C}$ in $5 \% \mathrm{CO}_{2}$. The shRNA plasmid of BMI-1 was purchased from the GeneChem Co. ECA109 and TE13 cells were plated in 12-well plates at a density of 300 cells/well in triplicates, and plasmid multiplicity of infection (MOI) was 10 . The cells were cultured in RPMI-1640 + 10\% FBS with $5 \mu \mathrm{g} / \mathrm{ml}$ Polybrene for $12 \mathrm{~h}$. Cells were collected $12 \mathrm{~h}$ after transduction with the plasmid and were selected in puromycin for stable clones. Subsequently, the cells were used for irradiation as indicated.

$X$-ray irradiation. Irradiation was performed at room temperature with a 6-MV Siemens linear accelerator (Siemens, Concord, CA, USA) at a dose rate of $2 \mathrm{~Gy} / \mathrm{min}$. After irradiation, the cells were recovered in an incubator for the indicated time until harvesting.

shRNA transfection. For the shRNA analyses, human BMI-1 small interfering RNA (shRNA) with the nucleotide sequence: 5'-GCUUAUCCAUUGAAUUCUUUGACCA-3' (sense) and 5'-UGGUCAAAGAAUUCAAUGGAUAAGC-3' (antisense), corresponding to part of the BMI-1 mRNA; and the negative control (NC) scrambled shRNA (NC-shRNA sense, 5'-UUCU CCGAACGUGUCACGUTT-3' and antisense, 5'-ACGUGACA GGUUCGGAGAATT-3') were designed and purchased from Invitrogen Corp. (Carlsbad, CA, USA). All of the shRNA sequences were subjected to the Basic Local Alignment Search Tool (BLAST) to confirm the absence of homology to any additional known coding sequences in the human genome. Cells were transfected using Lipofectamine RNAiMAX (Invitrogen) according to the manufacturer's protocol. Briefly, one day prior to transfection, ECA109 and TE13 cells ( $5 \times 10^{5} /$ well) were cultured in 6 -well tissue culture plates until they reached $50 \%$ confluency, and then the cells were transiently transfected with either BMI-1-shRNA or NC-shRNA (100 nM).

Quantitative real-time reverse transcription-polymerase chain reaction ( $q R T-P C R)$. Total RNAs were extracted using TRIzol reagent. Real-time PCR was then performed using Platinum SYBR-Green qPCR SuperMix-UDG (Invitrogen) according to the manufacturer's protocol. Briefly, after the reverse transcription reaction at $42^{\circ} \mathrm{C}$ for $60 \mathrm{~min}$ and $70^{\circ} \mathrm{C}$ for 5 min, cDNAs were synthesized using the ReverAid First Strand cDNA Synthesis kit (Thermo, Waltham, MA, USA), and then initially denatured at $94^{\circ} \mathrm{C}$ for $30 \mathrm{sec}$, followed by 40 cycles of the repeated procedure as follows: denaturation at $94^{\circ} \mathrm{C}$ for $5 \mathrm{sec}$, annealing at $56^{\circ} \mathrm{C}$ for $15 \mathrm{sec}$ and extension at $72^{\circ} \mathrm{C}$ for $10 \mathrm{sec}$. As a control, the levels of glyceraldehyde3-phosphate dehydrogenase (GAPDH) expression were also analyzed. Incorporation of the SYBR-Green dye into PCR products was monitored in real-time with LightCycler real-time PCR detection system (Roche Applied Science, Indianapolis, IN, USA), thereby allowing determination of the threshold cycle $(\mathrm{Ct})$ at which exponential amplification of products begins. Independent experiments were repeated 3 times for each sample, and the relative expression levels of genes were analyzed using the $2^{-\Delta \Delta \mathrm{Ct}}$ method (13).

Western blot analysis. The cellular total protein was solubilized in RIPA lysis buffer (1\% Triton X-100, $150 \mathrm{mM} \mathrm{NaCl}$, 10 mM Tris-HCl, pH 7.4, 1 mM EDTA, 1 mM EGTA, pH 8.0, $0.2 \mathrm{mM} \mathrm{Na}_{3} \mathrm{VO}_{4}, 0.2 \mathrm{mM}$ phenylmethylsulfonyl fluoride and $0.5 \% \mathrm{NP}-40)$. The protein amount was evaluated using BCA assays and separated on $10 \%$ SDS-PAGE gel, and electrotransferred to polyvinylidene fluoride (PVDF) membranes (Pierce, Rockford, IL, USA). The membranes were incubated overnight at $4^{\circ} \mathrm{C}$ with the indicated antibodies. The specific antibodies were rabbit BMI-1 (1:10,000), anti-p16 (1:50,00), anti-rH2AX (1:10,000), rabbit bcl-2 (1:1,000), anti-bax (1:500) and rabbit $\beta$-actin antibodies $(1: 10,000)$ (all from Abcam, Cambridge, MA, USA). After washing for $3 \times 5$ min with TBS-T, the membranes were incubated with secondary anti-rabbit IgG for $1 \mathrm{~h}$ at room temperature away from light. The membranes were scanned for the relative value of protein expression in gray scale by Image-Pro Plus software 6.0 (Media Cybernetics, Sliver Spring, MD, USA). The levels of the protein were calculated as the ratio of the intensity of protein to that of $\beta$-actin. Experiments were carried out in triplicate wells/time period and repeated 3 times.

Cell proliferation assay. The cells ( $2 \times 10^{3} /$ well) were cultured in 96-well tissue culture plates until they reached $50 \%$ confluency, and then transfected with a final concentration of $100 \mathrm{nM}$. After transfection for $24 \mathrm{~h}$, viability of the cells was determined at 24, 48 and $72 \mathrm{~h}$ after irradiation using the CellTiter 96 $\mathrm{A}_{\text {Queous }}$ One Solution cell proliferation assay (MTS) (Dojindo Molecular Technologies, Gaithersburg, MD, USA). Briefly, the cells were collected and incubated in medium containing $5 \mathrm{mg} / \mathrm{ml}$ MTS reagent (Promega Corporation, Madison, WI, USA) at $37^{\circ} \mathrm{C}$ for $2 \mathrm{~h}$. The absorbance at $492 \mathrm{~nm}$ was measured by an enzyme-linked immunosorbent assay (ELISA) plate reader. This experiment was repeated 3 times.

Transwell assay. Cell invasion was estimated using 24-well Transwell chambers with polycarbonate filters of $8-\mu \mathrm{m}$ pore size (Costar), and the chambers were precoated with extracellular matrix gel obtained from Corning (Corning, NY, USA). Twenty-four hours after transfection and $1 \mathrm{~h}$ after irradiation, an aliquot of $5 \times 10^{4}$ cells was placed in the upper chamber with $0.2 \mathrm{ml}$ serum-free medium, whereas the lower chamber (24-well plate) was loaded with $0.5 \mathrm{ml}$ of medium containing $10 \%$ FBS. The chambers were incubated in a humid atmo- 
A

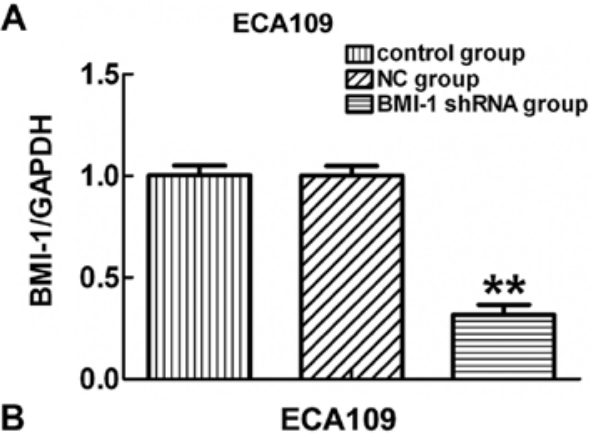

B
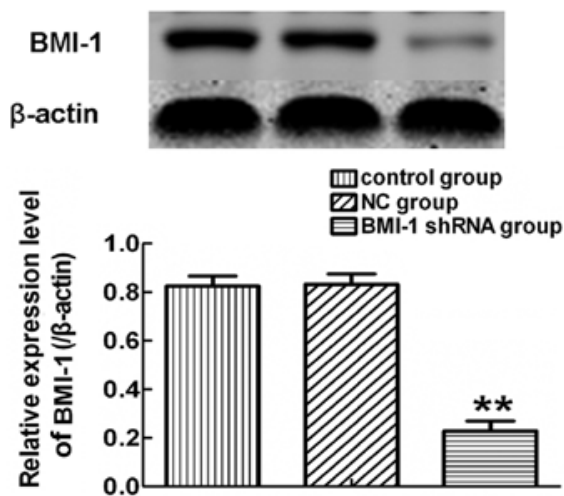

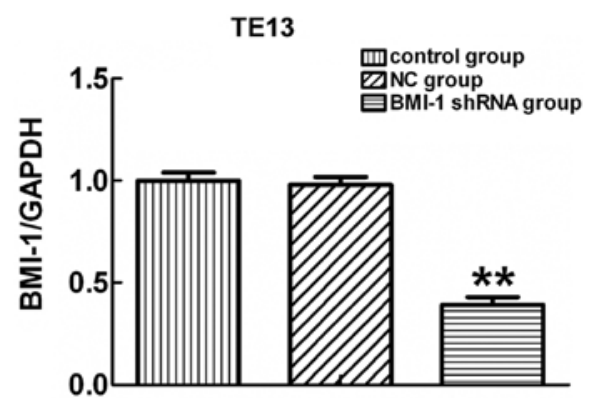

TE13

BMI-1

$\beta$-actin
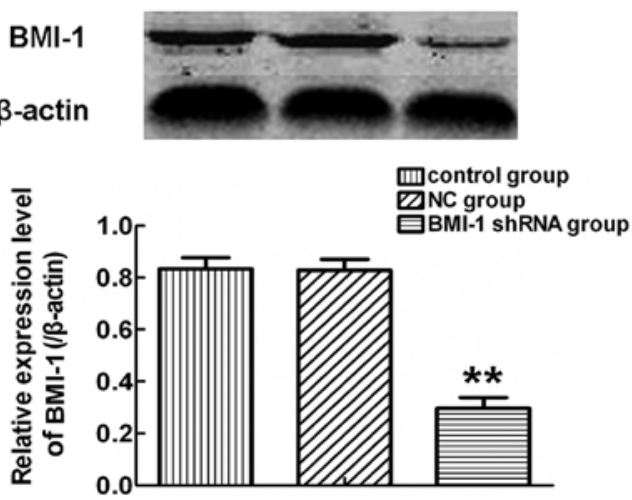

Figure 1. Downregulation of BMI-1 expression in the ECA109 and TE13 cells by shRNA. (A) qRT-PCR analysis showed that the mRNA expression levels of BMI-1 in the ECA109 and TE13 cells were significantly downregulated after transfection for $24 \mathrm{~h}$. (B) Similar results were found in the western blot analysis. ${ }^{* *} \mathrm{p}<0.01$ compared with the control and NC group.

sphere of $5 \% \mathrm{CO}_{2}$ at $37^{\circ} \mathrm{C}$ for $4.5 \mathrm{~h}$ in the invasion assay. After incubation, the cells on the upper surface of each filter were wiped off with a cotton swab. The cells on the lower surface of the filter were fixed with $4 \%$ formaldehyde, stained with crystal violet for $8 \mathrm{~min}$, and then washed with water. For each filter, the number of migrated or invaded cells in five fields (magnification, $\mathrm{x} 200$ ) was observed and counted.

Co-immunoprecipitation (Co-IP) assay. Cell lysates were first pre-cleared with $25 \mathrm{ml}$ of protein A-agarose (Santa Cruz Biotechnology, Santa Cruz, CA, USA). The supernatants were immunoprecipitated with $2 \mathrm{mg}$ of anti-rH2AX (1:100), anti-H2AK119ub (1:100), anti-BMI-1 (1:100) (all from Abcam) antibody for $2 \mathrm{~h}$ at $4^{\circ} \mathrm{C}$, followed by incubation with protein A-agarose overnight at $4^{\circ} \mathrm{C}$. Protein A-agarose antigen-antibody complexes were pelleted by centrifugation at $12,000 \mathrm{x} \mathrm{g}$ for $60 \mathrm{sec}$ at $4^{\circ} \mathrm{C}$. The pellets were washed five times with $1 \mathrm{ml}$ IPH buffer, for $20 \mathrm{~min}$ each time at $4^{\circ} \mathrm{C}$. Bound proteins were resolved by SDS-PAGE, followed by western blotting with antibodies against rH2AX, H2AK119ub and BMI-1.

Analysis of cell cycle distribution and apoptosis. Both cell cycle distribution and spontaneous apoptosis events were detected using a FACsCalibur II sorter and CellQuest FACS system (BD Biosciences, San Jose, CA, USA). To analyze cell cycle distribution, the cells were transfected using shRNA for $24 \mathrm{~h}$ and stimulated with irradiation for $24 \mathrm{~h}$ before being harvested. Cells were fixed with $70 \%$ ethanol at $4^{\circ} \mathrm{C}$ overnight, washed twice with phosphate-buffered saline (PBS) and resuspended in staining solution $(50 \mu \mathrm{g} / \mathrm{ml}$ propidium iodide, $1 \mathrm{mg} / \mathrm{ml} \mathrm{RNase} \mathrm{A,} 0.1 \%$ Triton X-100 in PBS) for $30 \mathrm{~min}$ at $37^{\circ} \mathrm{C}$ in the dark. To detect the extent of apoptosis under stress conditions, cells were transfected using shRNA for $24 \mathrm{~h}$ before irradiation and stained with fluorescein isothiocyanate (FITC)-conjugated Annexin V and 7-amino-actinomycin D (7-AAD) using the Annexin V-FITC apoptosis detection kit (BD Pharmingen) according to the manufacturer's protocol.

Colony formation assay. Cell survival curves were generated by a standard colony formation assay with minor modifications (14). Precooled tumor cells were irradiated by graded single doses (0-8 Gy) in control, NC and BMI-1 shRNA groups, seeded in Petri dishes, and cultivated in RPMI-1640 supplemented with $10 \%$ FBS. The experiments were repeated at least twice. Two weeks later, the cells were fixed and stained with crystal violet $(0.6 \%)$. Colonies exceeding 50 cells were scored as survivors.

Statistical analysis. Statistical analysis was conducted with the SPSS software package version 13.0 (SPSS, Inc., Chicago, IL, USA). All data are presented as the mean \pm standard deviation (SD), and analyzed using ANOVA with SPSS 13.0. For all tests, a p-value of $<0.05$ and 0.01 was considered to be statistically significant and is indicated by asterisks in the figures. All p-values given are the results of two-sided tests. Data were obtained from at least three independent experiments with a similar pattern.

\section{Results}

shRNAs targeting the BMI-1 gene and protein downregulate BMI-1 expression in the ECA109 and TE13 cells following transfection. To address the functional importance of the BMI-1 gene, we employed shRNA to deplete its expression 
A
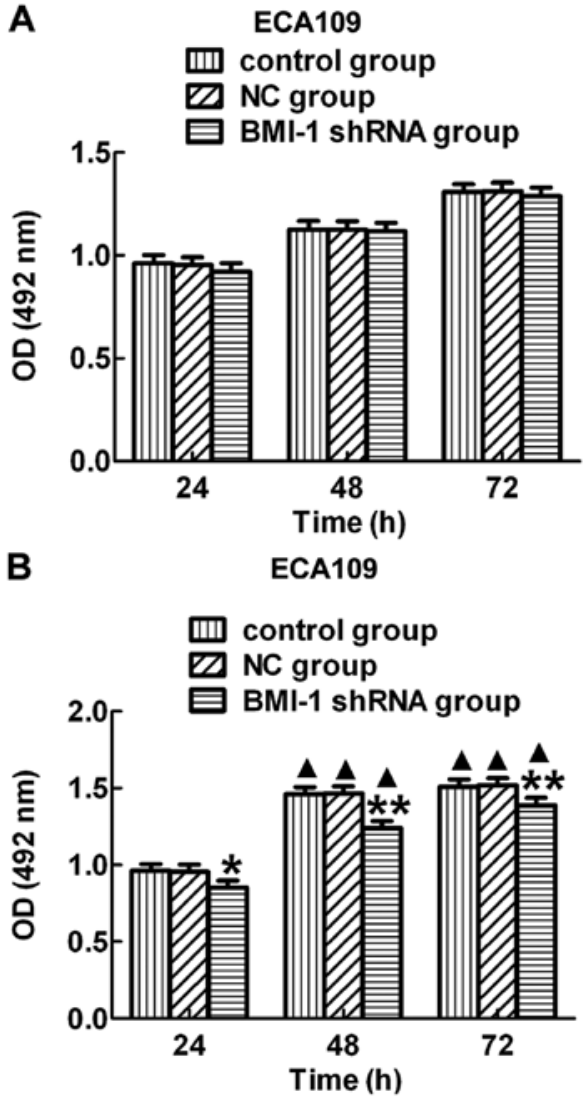

C

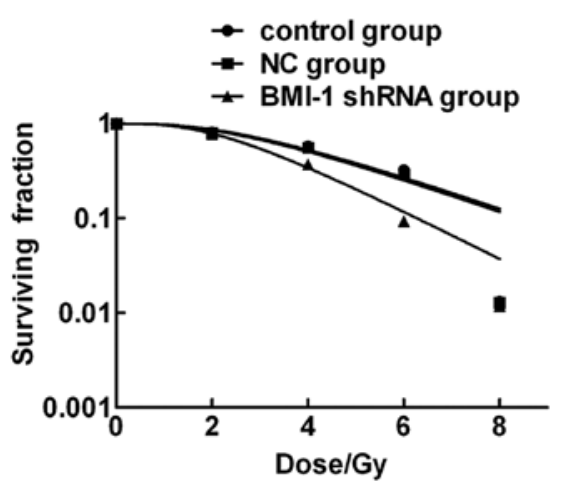

TE13

control group

NC group

BMI-1 shRNA group
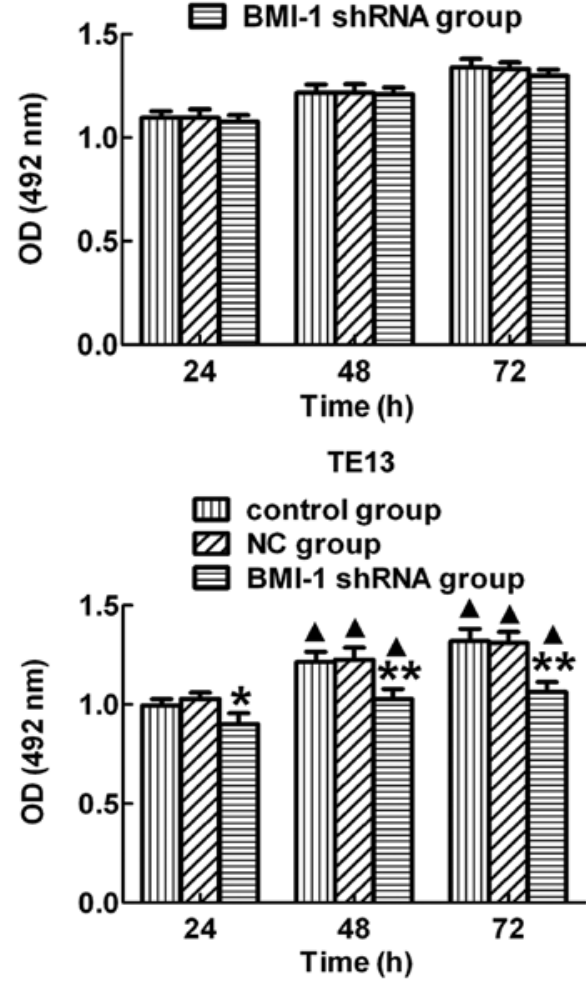

TE13

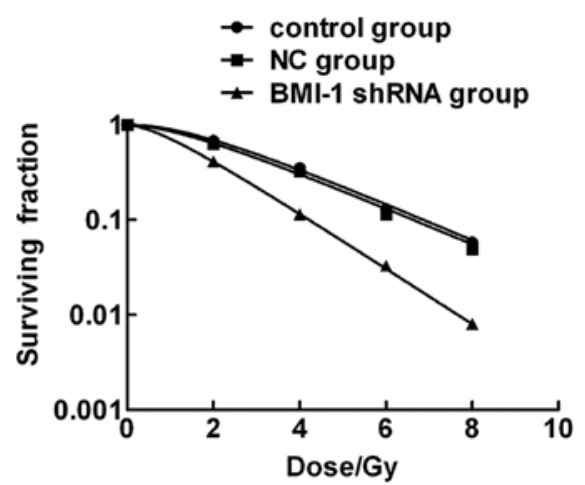

Figure 2. Effect of BMI-1 shRNA on the proliferation of ECA109 and TE13 cells after transfection and at 24, 48 and $72 \mathrm{~h}$ with irradiation or not. (A) There were no obvious differences among the control, NC and BMI-1 shRNA group without irradiation both in the ECA109 and TE13 cell lines, while (B) downregulation of BMI-1 expression by BMI-1 shRNA significantly inhibited the growth of ECA109 and TE13 cells after irradiation. Moreover, the proliferation levels in the 3 groups at 48 and $72 \mathrm{~h}$ after IR were higher than before irradiation. (C) Survival curves of the cells following IR are shown, respectively. GraphPad Prism 5.0 was used to fit cell survival curves, as well as the radiation biological parameters. The cells of the control and NC group had a broader shoulder compared to the BMI-1 shRNA group cells, indicating that they were more radioresistant than the latter. ${ }^{*} \mathrm{p}<0.05,{ }^{* *} \mathrm{p}<0.01$ compared with the control and $\mathrm{NC}$ group; ${ }^{\mathrm{p}} \mathrm{p}<0.05$ compared with the corresponding unirradiated group.

in ECA109 and TE13 cells, both of which were treated with NC-shRNA or shRNA targeting the BMI-1 gene. After transfection for $24 \mathrm{~h}$, the expression of BMI-1 in cells was examined by real-time quantitative reverse transcriptionpolymerase chain reaction (qRT-PCR) and western blot analysis. The qRT-PCR analysis confirmed that the levels of glyceraldehyde-3-phosphate dehydrogenase (GAPDH) were unaffected by transfection of BMI-1-shRNA or NC-shRNA. As shown in Fig. 1A, qRT-PCR showed that the expression of BMI-1 in the BMI-1-shRNA group was significantly lower than the levels in the control and NC-shRNA group after transfection for $24 \mathrm{~h}(\mathrm{p}<0.01)$. Similar results were observed in the western blot analysis (Fig. 1B). These data indicated that BMI-1-specific shRNA effectively and obviously suppressed the expression of BMI-1 in the ECA109 and TE13 cells.

Specific knockdown of BMI-1 expression by shRNA inhibits the growth and improves the radiosensitivity of ECA109 and TE13 cells after IR in vitro. To evaluate the effect of BMI-1 on ECA109 and TE13 cell proliferation, cells in the 3 groups were harvested at $24 \mathrm{~h}$ after transfection with shRNA of BMI- 1 and at 24,48 and $72 \mathrm{~h}$ after irradiation. MTS assay results showed that the proliferation rates of the ECA109 and TE13 cells were not significantly different in the 3 groups at each time point without irradia- 
A
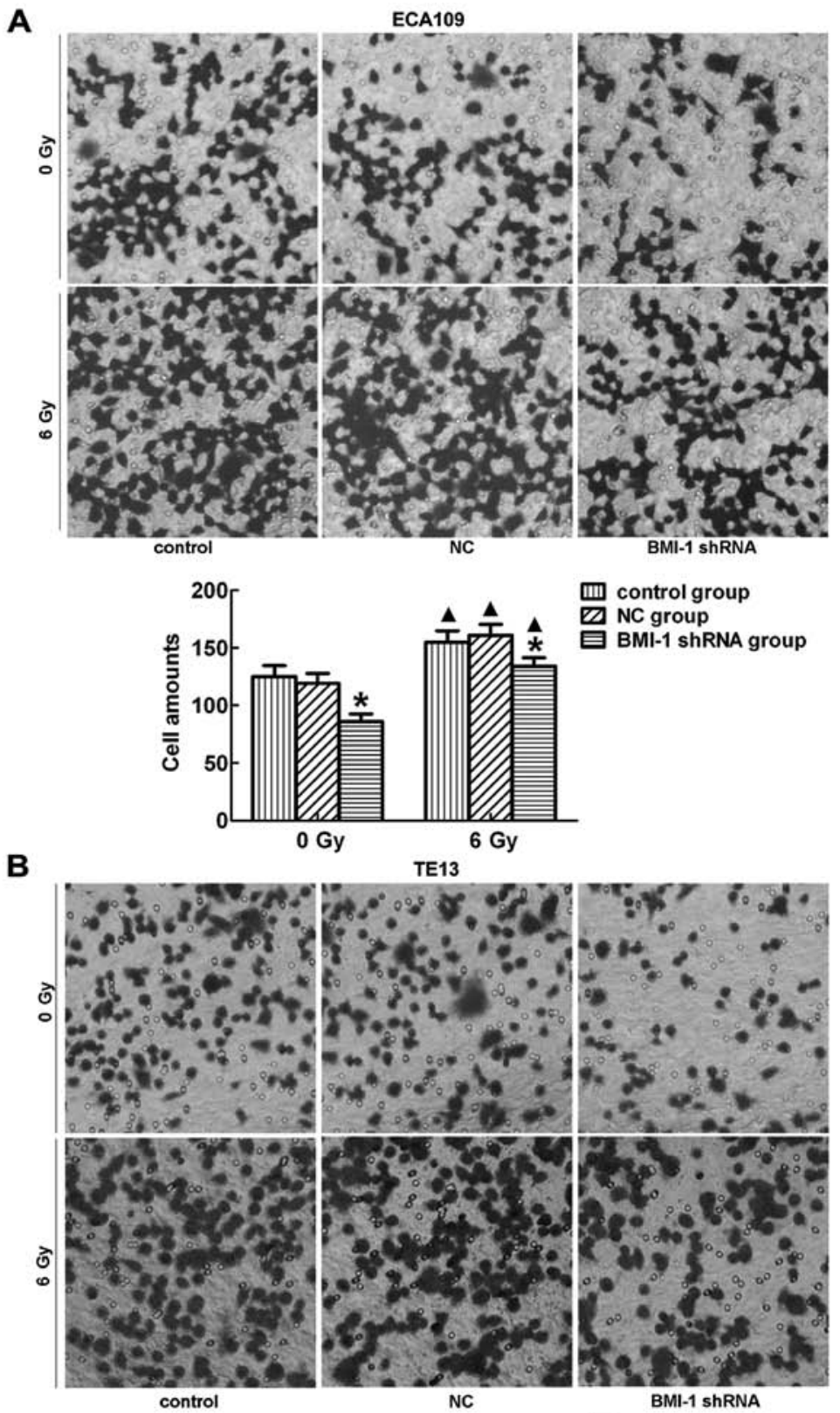

TE13
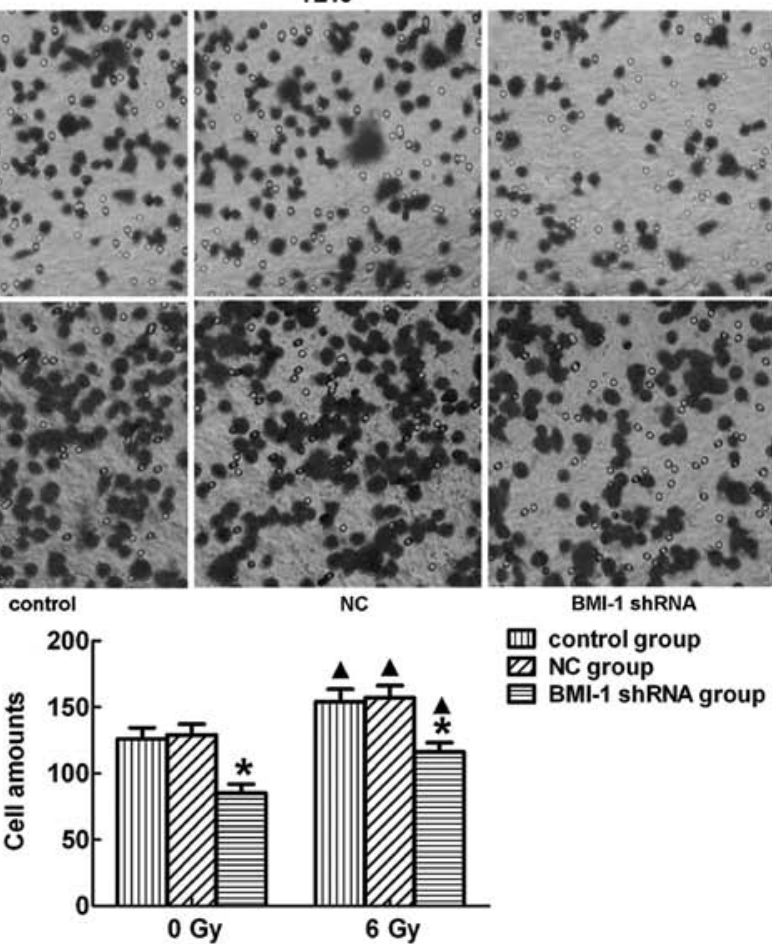

皿 control group

U NC group

目 BMI-1 shRNA group

Figure 3. Assessment of ECA109 and TE13 cell invasion. Invading cells were stained with crystal violet and visualized by microscopy (original magnification, $\mathrm{x} 200$ ). Transwell assay indicates that BMI-1 shRNA significantly reduced the invasion of esophageal cancer cells before and after irradiation (A, ECA109; $\mathrm{B}, \mathrm{TE} 13)$. Data are presented as the mean $\pm \mathrm{SD}$. ${ }^{*} \mathrm{p}<0.05$ compared with the control and $\mathrm{NC}$ group; ${ }^{\star} \mathrm{p}<0.05$ compared with the corresponding unirradiated group.

tion (Fig. 2A). However, the proliferation levels of the ECA109 and TE13 cells were significantly lower in the BMI-1 shRNA group when compared with the proliferation levels noted in the control and NC-shRNA groups after IR (Fig. 2B). Moreover, the proliferation levels in each group were obviously higher at 48 and $72 \mathrm{~h}$ after IR than these levels in the corresponding unirradiated groups. In addition, we also detected the radiation sensitivity in the different groups after exposure to IR and found that there was no significant difference between the control and $\mathrm{NC}$ groups. However, cells in the BMI-1 shRNA group had higher sensitivity than that noted in the other groups (Fig. 2C). These results suggest that downregulation of BMI-1 after irradiation significantly inhibited the proliferation of the ECA109 and TE13 cells and increased radiation sensitivity. 
A

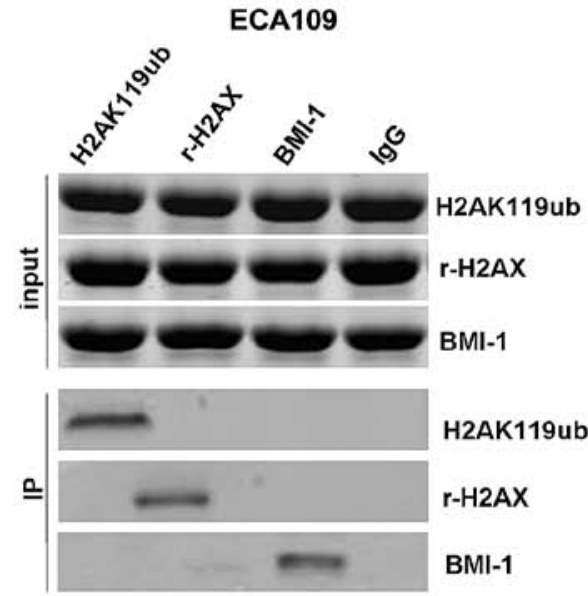

B

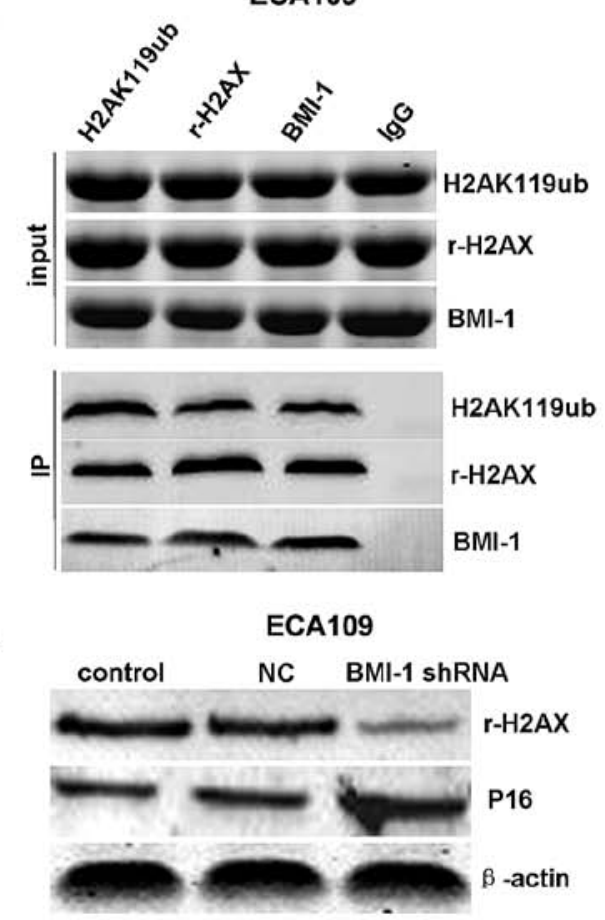

TE13
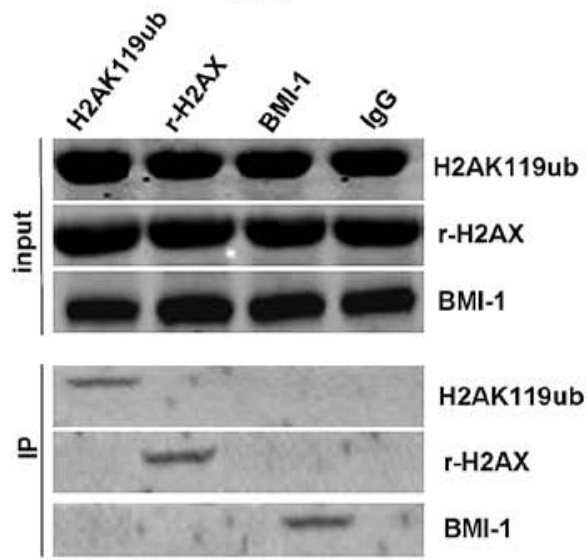

TE13
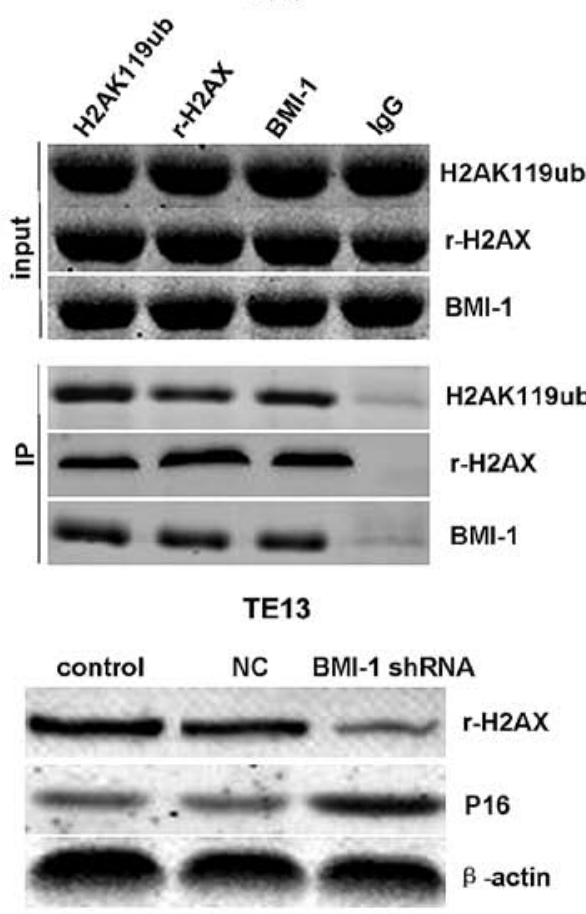

Figure 4. Interaction of BMI-1, H2AK119ub and r-H2AX, in ECA109 and TE13 esophageal cancer cells after irradiation or not. (A) There was no significant interaction without irradiation in the cells, while (B) the interaction between BMI-1 and H2AK119ub or r-H2AX was obviously enhanced in cells after irradiation, implying that BMI-1 may be related to the phosphorylation and ubiquitination of H2AX. (C) In addition, downregulation of r-H2AX and upregulation of P16 were detected in the BMI-1 shRNA group after knockdown of BMI-1 expression.

BMI-1 shRNA inhibits the invasive ability of the ECA109 and TE13 cells. Transwell assay revealed that the ECA109 and TE13 cells transfected with BMI-1 shRNA had a much lower invasive activity than that noted in the control and $\mathrm{NC}$ group cells $(\mathrm{p}<0.05)$ before and after irradiation (Fig. 3A, ECA109; and B, TE13). This suggests that suppression of BMI-1 had an inhibitory effect on the invasion of cells. There was no significant difference between the control and NC group.

BMI-1 interacts with the ubiquitination and phosphorylation of H2AX in ECA109 and TE13 cells in a DNA damage-induced manner. The specificity of this interaction was confirmed by Co-IP of H2AK119ub, r-H2AX and BMI-1. Notably, the interactions between BMI-1 and H2AK119ub or r-H2AX were obviously enhanced in the ECA109 and TE13 cells after
IR (Fig. 4B), while there was no significant interaction without irradiation (Fig. 4A). This modification was induced by DNA damage as we observed that the ubiquitination and phosphorylation of $\mathrm{H} 2 \mathrm{AX}$ were increased upon IR, indicating that BMI-1 may play an important role in DNA damage repair through inducing the ubiquitination and phosphorylation of H2AX. In addition, the downregulation of $\mathrm{r}-\mathrm{H} 2 \mathrm{AX}$ and upregulation of P16 were detected in the BMI-1 shRNA group after knockdown of BMI-1 expression (Fig. 4C).

Mechanisms of radiosensitization of the silencing of BMI-1. In order to further explore the mechanisms involved in the promotion of radiosensitization of esophageal cancer cells by silencing of BMI-1, flow cytometry was performed and demonstrated that irradiation obviously induced cell cycle 
A

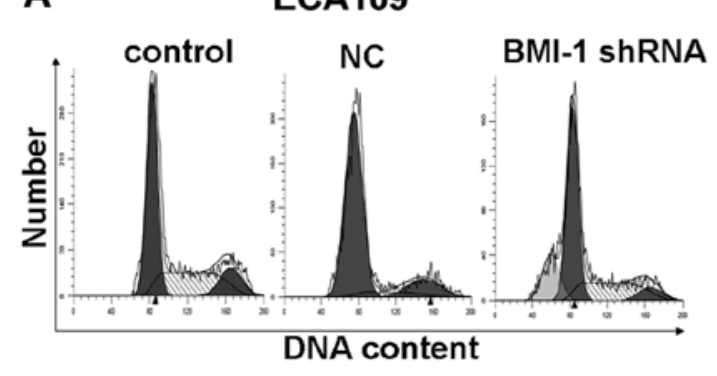

B

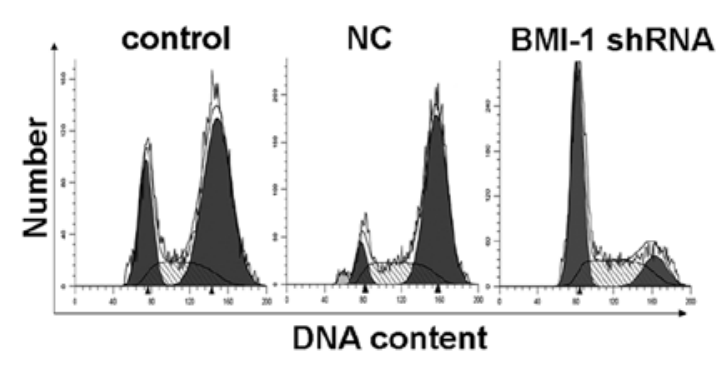

C

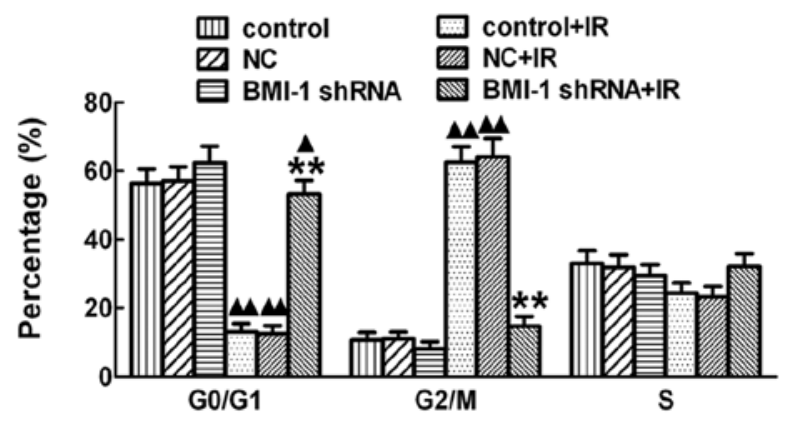

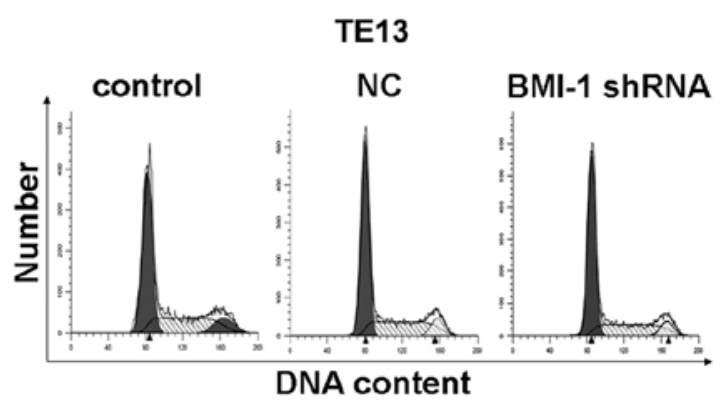

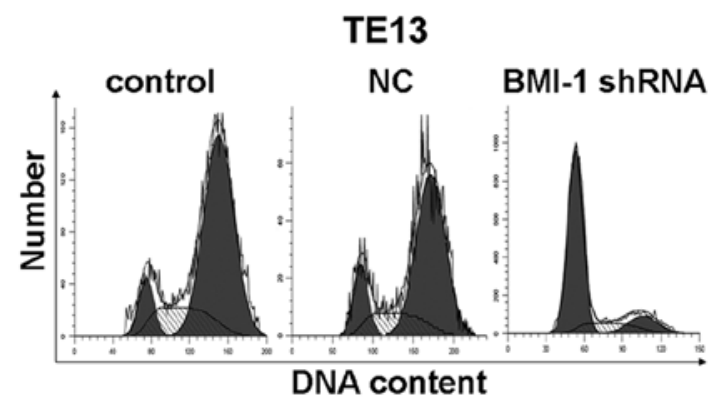

TE13

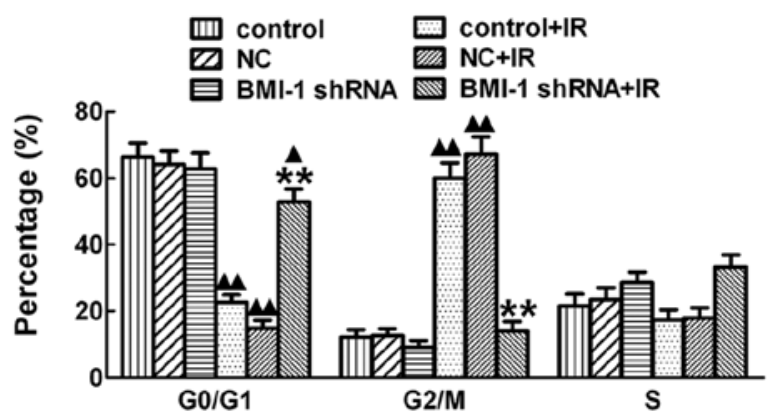

Figure 5. BMI-1 shRNA eliminates IR-induced cell cycle arrest in the ECA109 and TE13 cells. ECA109 and TE13 cells were pretreated with shRNA for $24 \mathrm{~h}$ and then irradiated with 6 Gy. At $24 \mathrm{~h}$ after IR, the cell cycle distribution was analyzed through FACS with 7-AAD staining. (A) Changes in the cell cycle in the control, NC and BMI-1 shRNA groups in the ECA109 and TE13 cells were not significantly different before IR. (B) However, the rates of cell cycle arrest at the G2/M phase were significantly higher after exposure to irradiation than before IR. In addition, the cell cycle arrest at G2/M stage was obviously weaken by silencing of the expression of BMI-1 after irradiation. (C) Data are shown as the means \pm standard error for each group before and after IR in the ECA109 and TE13 cells. ${ }^{* *} \mathrm{p}<0.01$ compared with the control and $\mathrm{NC}$ group; ${ }^{\wedge} \mathrm{p}<0.05,{ }^{\wedge} \mathrm{p}<<0.01$ compared with the corresponding unirradiated group.

arrest at the G2/M stage after irradiation for $24 \mathrm{~h}$ both in the ECA109 and TE13 cells in vitro. However, silencing of BMI-1 by shRNA obviously decreased the cell cycle arrest after irradiation (Fig. 5B), which allowed much time for repair of damaged tumor cells. Cancer cell repair may decrease the killing effect of RT, which induces radioresistance. With no irradiation, there was no significant difference among the BMI-1 shRNA, control and NC groups (Fig. 5A). Our results showed that knockdown of BMI-1 expression in esophageal cancer ECA109 and TE13 cells by RNA interference technology after exposure to irradiation decreased the G2/M phase arrest in cells to a certain degree, reducing the opportunity of tumor cells to repair thereby enhancing radiosensitivity.

In addition, the apoptosis rate was not obviously different among the control, NC and BMI-1 shRNA groups, in vitro without irradiation (Fig. 6A). However, the apoptosis rate in the BMI-1 shRNA group was significantly higher than that of the other two groups after RT both in the ECA109 and TE13 cells (Fig. 6B and C). In addition, the apoptosis levels in the different groups were increased after irradiation. In order to further explore the mechanism, we observed the expression levels of bcl-2 and bax after (Fig. 7B) and before irradiation (Fig. 7A). Significant downregulation of bcl-2 (Fig. 7C) and upregulation of bax (Fig. 7D) were observed in the BMI-1shRNA group after treatment with irradiation. Notably, there were no significant changes among the 3 groups without irradiation. Thus, BMI-1 has the ability to regulate the cell cycle, induce apoptosis and thus promote radiosensitivity after IR.

\section{Discussion}

Esophageal carcinoma is one of the most frequent malignant tumors, and is associated with the highest morbidity and mortality worldwide. For example, the incidence of esophageal carcinoma is as high as 26.3/100,000 in the US, which is higher than that in Asia. Radiotherapy (RT) is one of the most important treatment methods for esophageal carcinoma, but the 5 -year survival rate is only 10 to $15 \%$, accompanied by 
A
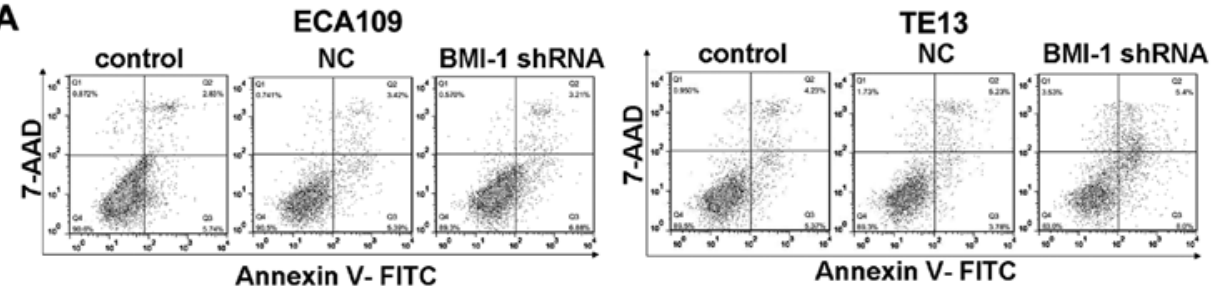

B

ECA109

TE13
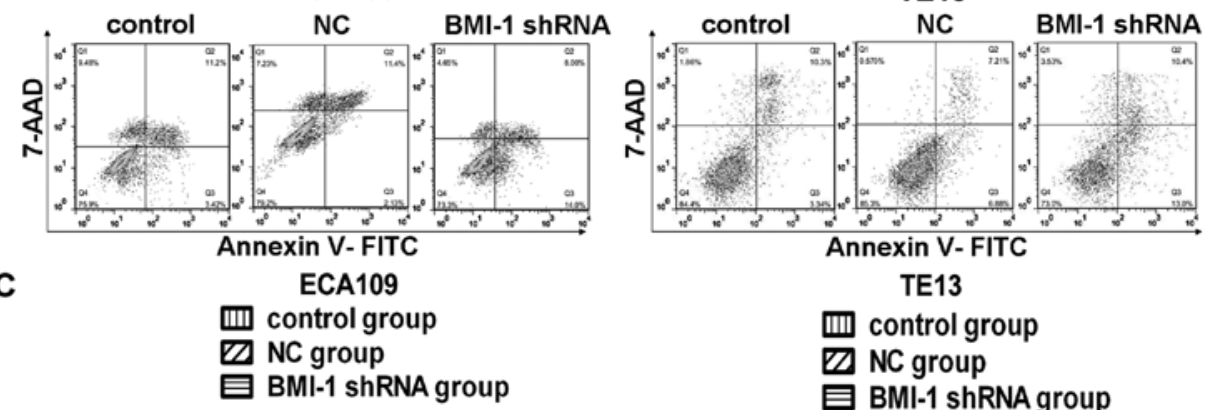

TE13

inf control group

$\square$ NC group

目 BMI-1 shRNA group
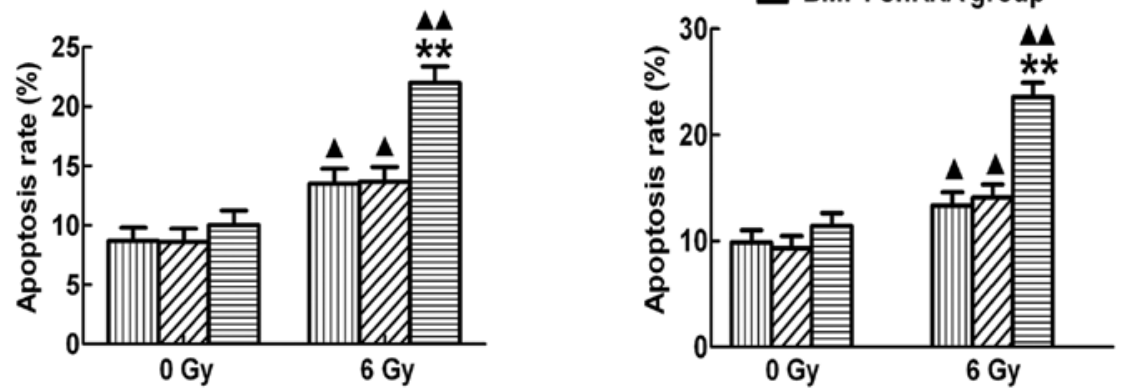

Figure 6. Apoptosis rate among the different groups in the ECA109 and TE13 cells in vitro. (A) The apoptosis rates of the control, NC and BMI-1 groups were not statistically different both in the ECA109 and TE13 cell lines before irradiation. (B) However, the apoptosis rates in the 3 groups were significantly different after exposure to irradiation in the cell lines and obviously higher than their corresponding groups before IR. In addition, the apoptosis rates were significantly higher in the BMI-1 shRNA group than those of the control and NC groups after exposure to irradiation. (C) Apoptosis rates are shown as the means \pm standard error for each group before and after IR. ${ }^{* *} \mathrm{p}<0.01$ compared with the control and NC group; ${ }^{\wedge} \mathrm{p}<0.05,{ }^{\wedge} \mathrm{p}<0.01$ compared with the corresponding unirradiated group.

a high recurrence rate at $\sim 60$ to $80 \%$. Despite improvement in the local control rate after extensive application of new techniques in RT, local failure is still the main cause of death. Moreover, the molecular mechanisms behind the development and progression of esophageal carcinoma after RT are unknown.

BMI-1, one of the core components in polycomb repressive complex 1, may play an important role in the immortalization of normal epithelial cells and early malignant transformation, as well as in the maintenance of the self-renewal of stem cells and carcinogenesis in esophageal carcinoma. In addition to their role in development, as one of the polycomb group proteins, BMI-1 has been reported to be overexpressed in a variety of human cancers, such as malignant lymphomas and various solid tumors (15). In the present study, we designed and constructed an shRNA recombinant expression vector targeting BMI-1 and detected its expression at both the protein and mRNA levels after transfection for $24 \mathrm{~h}$. The findings demonstrated that BMI-1 expression at both the protein and gene levels was obviously lower in the BMI-1-shRNA group than those noted in the control and the NC groups, while there was no significant difference between the control and the NC group, indicating that the shRNA targeting of BMI-1 decreased the BMI-1 expression, and may be a potential therapeutic strategy for the gene therapy of esophageal carcinoma.
In the present study, we also investigated the effects on proliferation, invasion and radiosensitivity of esophageal carcinoma cells by BMI-1 shRNA following irradiation. The suppression of BMI-1 expression in the ECA109 and TE13 cells significantly inhibited cell proliferation and improved radiosensitivity after the cells were treated with shRNA and irradiation at different times. The invasive ability of the cancer cells in the BMI-1 shRNA group was also obviously suppressed after irradiation or not. Previous studies have reported that the downregulation of BMI-1 by adenovirus-mediated delivery of BMI-1 shRNA reduced the proliferation and invasion of cancer cells and decreased the radioresistance $(16,17)$. Notably, there were no obvious differences among the control group, NC and BMI-1-shRNA group without irradiation either in proliferation or in radiosensitivity, indicating that RT combined with shRNA effectively inhibits the proliferation of tumor cells and enhances radiosensitization.

It was reported that BMI-1 is related to the ubiquitination and phosphorylation of H2AX (18). Recent studies identified that BMI-1 possesses E3 ligase activity for $\mathrm{H} 2 \mathrm{AX}$ monoubiquitination and interacts with $\mathrm{H} 2 \mathrm{AX}$ in a DNA damage-induced manner (19). In accordance with previous research, our results found that interaction between BMI-1 and ub-H2AX or $\mathrm{r}-\mathrm{H} 2 \mathrm{AX}$ was obviously enhanced in esophageal carcinoma after exposure to IR, while there was no significant interaction 
A
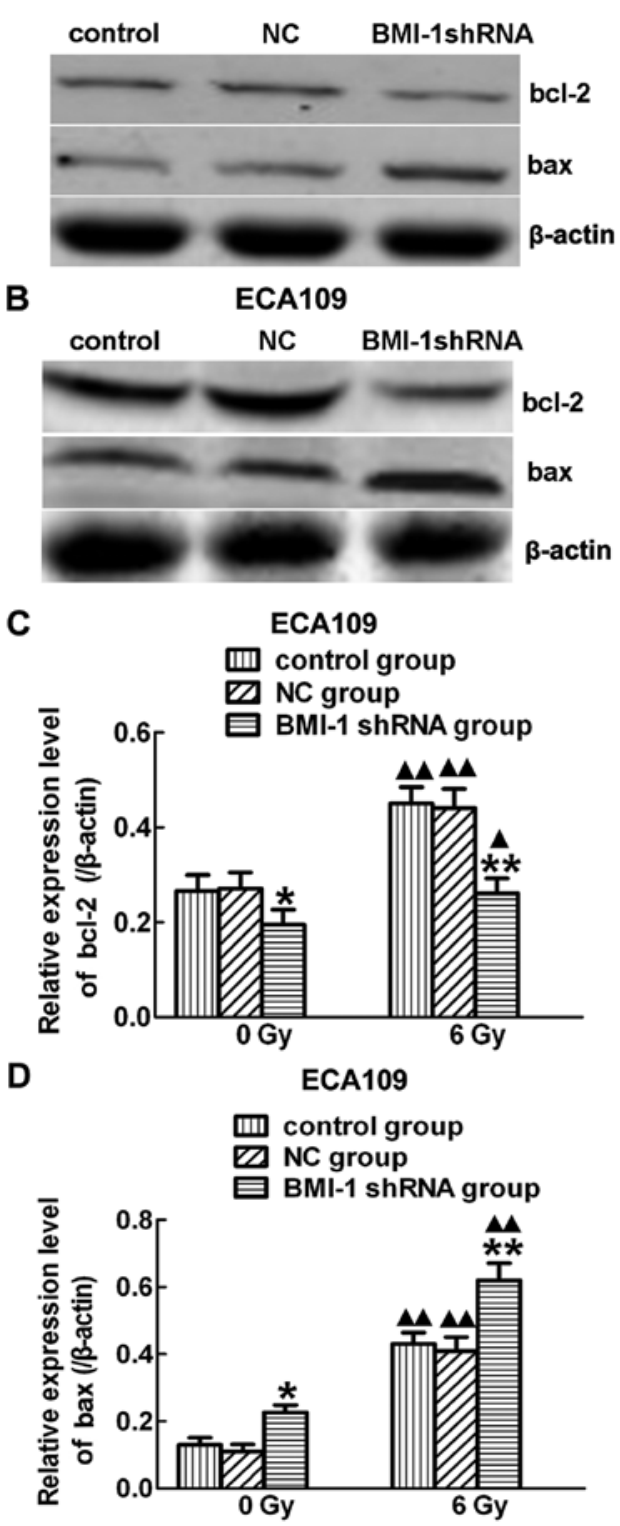

TE13

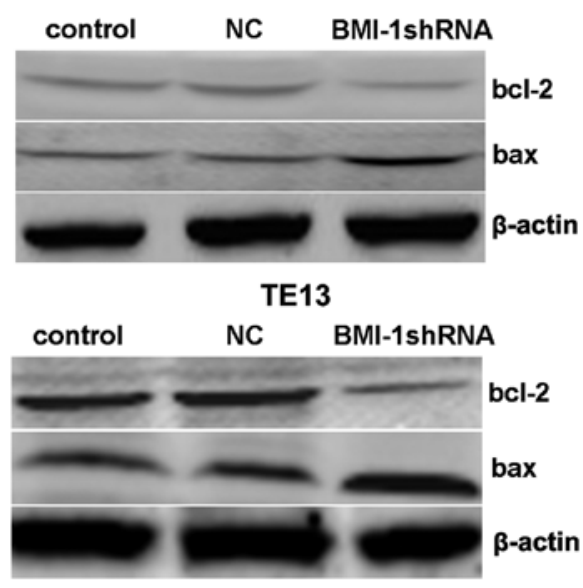

TE13
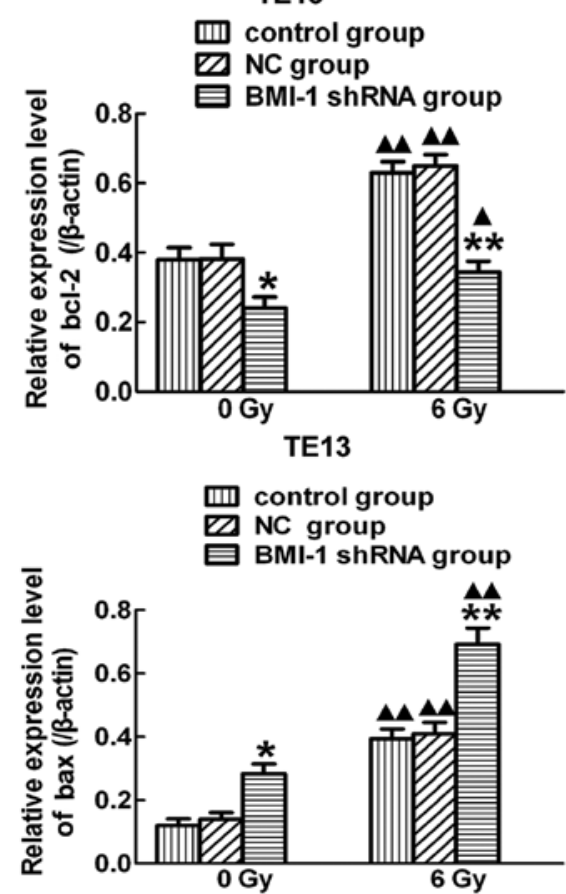

Figure 7. Protein expression is associated with apoptosis among the different groups in the ECA109 and TE13 cells in vitro. The downregulation of bcl-2 and upregulation of bax in ECA109 and TE13 cells was significantly observed (A) before and (B) after exposure to irradiation. In addition, the expression levels of bcl-2 and bax after irradiation in the 3 groups were higher than before IR. (C) Histogram of the bcl-2 level is shown as the means \pm standard error for each group before and after IR. (D) Histogram of the bax level is presented as the means \pm standard error for each group before and after IR. ${ }^{*} \mathrm{p}<0.05$, ${ }^{* *} \mathrm{p}<0.01 \mathrm{compared}$ with the control and $\mathrm{NC}$ group; ${ }^{\wedge} \mathrm{p}<0.05,{ }^{\wedge} \mathrm{p}<0.01$ compared with the corresponding unirradiated group.

without irradiation, indicating a vital role of BMI-1 in DDR and its potential mechanism. Our pre-trial confirmed that it could increase radiation sensitivity through knockdown of $\mathrm{H} 2 \mathrm{AX}$ in esophageal cancer and cell cycle arrest at the G0/ G1 phase (12). In the present study, we found that silencing of the BMI-1 gene by shRNA significantly decreased the phosphorylation of $\mathrm{H} 2 \mathrm{AX}$, followed by upregulation of $\mathrm{p} 16$, which is able to inactivate CDK by directly binding to CDK4 and CDK6, leading to cell cycle arrest (20). These data indicated that BMI-1 may play an important role in regulating the cell cycle through interaction with $\mathrm{H} 2 \mathrm{AX}$.

Cellular responses to DNA damage include cell cycle arrest and apoptosis. The highest expression of BMI-1 is observed in the $\mathrm{G} 2 / \mathrm{M}$ phase $(21,22)$. Our data revealed that inhibition of the expression of BMI-1 by shRNA in ECA109 and TE13 cells significantly induced spontaneous cell apoptosis and eliminated cell cycle arrest at the G2/M phase of the cell cycle after RT. However, given no ionizing radiation, there was no statistically significant difference among the control, $\mathrm{NC}$ and BMI-1 shRNA group. These results are in accordance with previous studies on esophageal carcinoma (23) and cervical carcinoma cells (24). Accordingly, this method of suppressing BMI-1 expression which leads to cell growth inhibition and the induction of apoptosis, in turn reduces the proliferation of esophageal carcinoma cells, indicating that it may have a significant therapeutic effect on esophageal carcinoma $(25,26)$. In the present study, our data showed that the apoptosis rate of cells was higher in the BMI-1 shRNA group than that noted in the control and NC group after exposure to irradiation, while there was no statistically significant difference among the 
3 groups without RT. Meanwhile, our results further indicated that the promotive effect of BMI-1 shRNA on the apoptosis in ECA109 and TE13 cells after treatment with irradiation was accompanied by upregulated expression of bcl-2 and downregulation of bax, which are associated with cell apoptosis after DNA damage. These results are in accordance with previous studies, which showed that BMI-1 increased cell apoptosis through regulation of apoptosis-related proteins (27-29), and further enhanced radiosensitivity.

In summary, our data explored the important role of BMI-1 in the growth and invasion of esophageal carcinoma by shRNA silencing of BMI-1 expression after IR or not. In addition, downregulation of BMI-1 decreased bcl-2 expression, enhanced bax expression and eliminated IR-induced G2/M arrest by inhibition of BMI-1 expression. To the best of our knowledge for the first time, we demonstrated the suppression of BMI-1 expression by shRNA after treatment with irradiation, indicating that BMI-1 may be an important gene for the gene targeted therapy of human esophageal carcinoma.

\section{Acknowledgements}

The present study was supported by the National Natural Science Foundation of China (no. 81372416) and the Medical Sciences Key Topics of Hebei Province (nos. 20160183 and 20150305).

\section{References}

1. Sun Y, Liu M, Yang B, Li B and Lu J: Role of siRNA silencing of MMP-2 gene on invasion and growth of laryngeal squamous cell carcinoma. Eur Arch Otorhinolaryngol 265: 1385-1391, 2008.

2. Zhao JX and Xie XL: Regulation of gene expression in laryngeal carcinama by microRNAs. Int J Pathol Clin Med 32: 222-225, 2012.

3. Nacerddine K, Beaudry JB, Ginjala V, Westerman B, Mattiroli F, Song JY, van der Poel H, Ponz OB, Pritchard C, Cornelissen-Steijger P, et al: Akt-mediated phosphorylation of Bmil modulates its oncogenic potential, E3 ligase activity, and DNA damage repair activity in mouse prostate cancer. J Clin Invest 122: 1920-1932, 2012.

4. Rouhigharabaei L, Ferreiro JF, Put N, Michaux L, Tousseyn T, Lefebvre C, Gardiner A, De Kelver W, Demuynck H, Verschuere $\mathrm{J}$, et al: $B M I 1$, the polycomb-group gene, is recurrently targeted by genomic rearrangements in progressive B-cell leukemia/lymphoma. Genes Chromosomes Cancer 52: 928-944, 2013.

5. Guo BH, Feng Y, Zhang R, Xu LH, Li MZ, Kung HF, Song LB and Zeng MS: Bmi-1 promotes invasion and metastasis, and its elevated expression is correlated with an advanced stage of breast cancer. Mol Cancer 10: 10, 2011.

6. Dong Q, Sharma S, Liu H, Chen L, Gu B, Sun X and Wang G: HDAC inhibitors reverse acquired radio resistance of KYSE-150R esophageal carcinoma cells by modulating Bmi-1 expression. Toxicol Lett 224: 121-129, 2014.

7. Chen Y, Lian G, Zhang Q, Zeng L, Qian C, Chen S and Huang K: Overexpression of Bmi-1 induces the malignant transformation of gastric epithelial cells in vitro. Oncol Res 21: 33-41, 2013.

8. Hoenerhoff MJ, Chu I, Barkan D, Liu ZY, Datta S, Dimri GP and Green JE: BMI1 cooperates with H-RAS to induce an aggressive breast cancer phenotype with brain metastases. Oncogene 28: 3022-3032, 2009.

9. Wang H, Wang L, Erdjument-Bromage H, Vidal M, Tempst P, Jones RS and Zhang Y: Role of histone H2A ubiquitination in Polycomb silencing. Nature 431: 873-878, 2004.
10. Buchwald G, van der Stoop P, Weichenrieder O, Perrakis A, van Lohuizen $M$ and Sixma TK: Structure and E3-ligase activity of the Ring-Ring complex of polycomb proteins Bmil and Ring1b. EMBO J 25: 2465-2474, 2006.

11. Cao R, Tsukada Y and Zhang Y: Role of Bmi-1 and Ring1A in H2A ubiquitylation and Hox gene silencing. Mol Cell 20: 845-854, 2005.

12. Shi HY and Zhu SC: Radiosensitization of esophageal cancer cells ECA109 by knockdown of H2AX. Thorac Cancer 4: 1759-7706, 2013.

13. Livak KJ and Schmittgen TD: Analysis of relative gene expression data using real-time quantitative PCR and the $2^{-\Delta \Delta C_{\mathrm{T}}}$ method. Methods 25: 402-408, 2001.

14. Bartek $\mathrm{J}$ and Lukas J: Chk1 and Chk2 kinases in checkpoint control and cancer. Cancer Cell 3: 421-429, 2003.

15. Raaphorst FM: Deregulated expression of Polycomb-group oncogenes in human malignant lymphomas and epithelial tumors. Hum Mol Genet 14 (Suppl 1): R93-R100, 2005.

16. Song W, Tao K, Li H, Jin C, Song Z, Li J, Shi H, Li X, Dang Z and Dou K: Bmi-1 is related to proliferation, survival and poor prognosis in pancreatic cancer. Cancer Sci 101: 1754-1760, 2010.

17. Liang W, Zhu D, Cui X, Su J, Liu H, Han J, Zhao F and Xie W: Knockdown BMI1 expression inhibits proliferation and invasion in human bladder cancer T24 cells. Mol Cell Biochem 382: 283-291, 2013.

18. Ginjala V, Nacerddine K, Kulkarni A, Oza J, Hill SJ, Yao M, Citterio E, van Lohuizen M and Ganesan S: BMI1 is recruited to DNA breaks and contributes to DNA damage-induced H2A ubiquitination and repair. Mol Cell Biol 31: 1972-1982, 2011.

19. Pan MR, Peng G, Hung WC and Lin SY: Monoubiquitination of $\mathrm{H} 2 \mathrm{AX}$ protein regulates DNA damage response signaling. J Biol Chem 286: 28599-28607, 2011

20. Suh HN and Han HJ: Collagen I regulates the self-renewal of mouse embryonic stem cells through $\alpha 2 \beta 1$ integrin- and DDR1dependent Bmi-1. J Cell Physiol 226: 3422-3432, 2011.

21. Xu Z, Liu H, Lv X, Liu Y, Li S and Li H: Knockdown of the Bmi-1 oncogene inhibits cell proliferation and induces cell apoptosis and is involved in the decrease of Akt phosphorylation in the human breast carcinoma cell line MCF-7. Oncol Rep 25: 409-418, 2011.

22. He X, Dong Y, Wu CW, Zhao Z, Ng SS, Chan FK, Sung JJ and Yu J: MicroRNA-218 inhibits cell cycle progression and promotes apoptosis in colon cancer by downregulating BMI1 polycomb ring finger oncogene. Mol Med 18: 1491-1498, 2013.

23. Liu WL, Guo XZ, Zhang LJ, Wang JY, Zhang G, Guan S, Chen YM, Kong QL, Xu LH, Li MZ, et al: Prognostic relevance of Bmi-1 expression and autoantibodies in esophageal squamous cell carcinoma. BMC Cancer 10: 467, 2010.

24. Min L, Shen D-X, Guo X-T, Guan T and Chen X-D: Clinicopathological and prognostic significance of Bmi-1 expression in human cervical cancer. Acta Obstet Gynecol Scand 90: 737-745, 2011.

25. Yao XB, Wang XX, Liu H, Zhang SQ and Zhu HL: Silencing Bmi-1 expression by RNA interference suppresses the growth of laryngeal carcinoma cells. Int J Mol Med 31: 1262-1272, 2013.

26. Rizo A, Olthof S, Han L, Vellenga E, de Haan G and Schuringa JJ: Repression of BMI1 in normal and leukemic human CD $34^{+}$ cells impairs self-renewal and induces apoptosis. Blood 114: 1498-1505, 2009.

27. Crea F, Duhagon Serrat MA, Hurt EM, Thomas SB, Danesi R and Farrar WL: BMI1 silencing enhances docetaxel activity and impairs antioxidant response in prostate cancer. Int J Cancer 128: 1946-1954, 2011.

28. Siddique HR, Parray A, Tarapore RS, Wang L, Mukhtar H, Karnes RJ, Deng Y, Konety BR and Saleem M: BMI1 polycomb group protein acts as a master switch for growth and death of tumor cells: Regulates TCF4-transcriptional factor-induced BCL2 signaling. PLoS One 8: e60664, 2013.

29. Zhu D, Wan X, Huang H, Chen X, Liang W, Zhao F, Lin T, Han J and Xie W: Knockdown of Bmil inhibits the stemness properties and tumorigenicity of human bladder cancer stem cell-like side population cells. Oncol Rep 31: 727-736, 2014. 